\title{
Nebelkarten der Schweiz
}

\begin{abstract}
Summary
The analysis of fog distribution over Switzerland consists of two different studies:

The first deals with the spatial determination of fog frequencies and fog types for the early morning. It was realized by using the daily observations of 320 stations. This point by point information was spatially interpolated with the aid of the information (mean fog cover frequency) of 94 typical fog distributions detected from NOAA satellite data. The derived fog map of Switzerland includes four different fog zones: ground fog zone, fog-free intermediary and high fog (stratus) zone, fog-free slope zone, lower up-slope fog zone.

The result of the second study consists of four maps showing the fog cover frequency of the important weather types with fog layers over the Swiss Alpine foreland. They were traced out by using the above mentioned satellite data. The four maps indicate clearly that the fog and cold air distribution during anticyclonic weather situations is strongly determined by the sub-synoptic pressure and wind field.
\end{abstract}

\section{Einleitung und Zielsetzung}

Der Nebel als Kondensationsprodukt der bodennahen Luftschicht ist oft an austauscharme Wetterlagen gebunden. Dieser Aufsatz befaßt sich vor allem mit diesem «Schönwetternebel», welcher bei antizyklonalen Druckverhältnissen auftritt und oft tagelang das Mittellandbekken und einzelne Alpentäler bedeckt. Solche Nebelvorkommen können sich lufthygienisch zu extremen Wettersituationen entwickeln, denn bei Boden-oder Hochnebel ist das für die Durchlüftung zur Verfügung stehende Luftvolumen vertikal stark eingeschränkt, und auch strahlungsbedingte Austauschprozesse treten nur bedingt auf. Nicht selten bilden sich eigentliche Smoglagen, in deren Verlauf sich die Luftschadstoffe der verschiedenen Emittenten (Straßenverkehr, Heizung, Industrie, Kehrrichtverbrennungsanlagen) anreichern.

Von besonderem Interesse ist die Ansammlung der Luftschadstoffe im Nebel. Im Vergleich zur Konzentration in Regentropfen kann die Konzentration in verdunstendem Nebel bis zu 100mal höher sein (STUMM et al., 1985). In den nebelreichen Zonen des Mittellandes und der Mittelgebirgslagen können diese hohen Konzentrationen möglicherweise biologische Schäden verursachen.

Der Nebel weist einerseits in bezug auf Häufigkeit und Intensität lokal große Unterschiede auf, und andererseits bietet er Probleme bei der Erfassung. Die Stationsinfor- mation (Nebelbeobachtung) hat nur in der näheren Umgebung des Beobachtungsortes Gültigkeit. Das gesamte Beobachtungsnetz liefert punktuelle Angaben über die Verteilung der Nebelhäufigkeit. Um das Ziel der Herstellung einer «Nebelkarte Schweiz» für den Klimaatlas zu erreichen, wurde noch eine flächendeckende Information benötigt, welche der Satellit lieferte. Die ganze Nebelkartierung Schweiz reduzierte sich vorerst auf ein methodisches Problem. Auf Grund des vorhandenen Datenmaterials wurden die beiden Verfahren der Nebelauswertung (Stations-, Satellitenbildauswertung) miteinander verbunden. Als Produkt entstand eine reine Nebelhäufigkeitskarte, die auch als Grundlage diente, um innerhalb der verschiedenen Nebelarten die Häufigkeiten anzugeben. Um einen aussagekräftigeren Detaillierungsgrad zu erhalten, erfolgte die eigentliche Nebelkartierung (Nebelart - Nebelhäufigkeit) im Maßstab $1: 200000$.

Eine raum-zeitliche Analyse der Nebelverhältnisse kann nur mit Hilfe von Satellitenbildern objektiv erfolgen. WANNER und KUNZ (1983) berechneten aus einem Kollektiv von 94 Satellitenbildern verschiedene Nebelbedeckungskarten. Nebst einer Mittelkarte der Nebelbedeckung wurden auch wetterlagenabhängige Nebelkarten erstellt. Die von WANNER und KUNZ (1983) berechnete Mittelkarte lieferte die oben erwähnte flächendeckende Information.

In dieser Arbeit werden demnach zwei Typen von Nebelkarten vorgestellt:

1. Mittelwertskarte Nebelart - Nebelhäufigkeit

$1: 1500000$

2. Wetterlagenabhängige Karten der Nebelbedeckung

Die gesamte Arbeit wurde unterstützt durch den schweizerischen Nationalfonds zur Förderung der wissenschaftlichen Forschung.

Franz Xaver Troxler, Geograph, Gymnasiallehrer, Wyttenbachstraße 11, CH-3013 Bern

Heinz Wanner, Prof. Dr. phil. nat., Geographisches Institut, Hallerstraße 12, CH-3012 Bern 


\section{Datengrundlage}

Laut Definition wird von Nebel gesprochen, wenn die horizontale Sichtweite weniger als $1000 \mathrm{~m}$ beträgt. Die Sichtverminderung wird durch in der Luft schwebende Wassertröpfchen hervorgerufen. Nebel kann demnach als eine dem Boden aufliegende Wolke bezeichnet werden. Die wichtigsten Datenquellen dieser Arbeit sind in Tabelle 1 zusammengestellt.

Tab.1: Datenmaterial

\author{
Symoptische stationen \\ Klimastationen \\ Regenmessstationen \\ Total
}

Satellitendaten in $2 \times 2 \mathrm{~km}^{2}$ Raster (verschiedene NOAA-Satellitenbilder)

Aus dieser Zusammenstellung wird ersichtlich, daß uns zwei ganz unterschiedliche Informationsquellen zur Verfügung standen: Auf der einen Seite die Stationsbeobachtungen (punktuelle Angaben), auf der andern Seite Satellitenbeobachtungen (flächendeckende Angaben).

\subsection{Stationsdaten}

Diese Nebeldaten wurden während fünf Winterhalbjahren (1970/71 bis 74/75, Monate Oktober bis März) besonders sorgfältig erhoben. Bis auf zwei Ausnahmen, Genf Cointrin und Zürich Flughafen, welche ein Transmissometer und somit ein objektives Maß der Nebelerfassung besitzen, sind sämtliche Nebeldaten Augenbeobachtungen. Die Beobachtung erfolgte durchwegs morgens zwischen 06 und 07 UTC. Trotz dieser Vereinheitlichung unterliegen die Daten subjektiven Einflüssen, die nur schwer auszumachen sind.

An dieser Stelle sei der Begriff «Nebeltag» definiert, wie er bei den Auswertungen verwendet wurde: Ein Tag wird dann als Nebeltag bezeichnet, wenn zur Beobachtungszeit zwischen 06 und 07 UTC die horizontale Sichtweite weniger als $1000 \mathrm{~m}$ beträgt.

Das zur Verfügung stehende Datenmaterial der 5jährigen Beobachtungsreihe darf als brauchbar angesehen werden, da einerseits $70 \%$ der Stationen eine gute Repräsentativität aufweisen und andererseits nicht allein auf die Stationswerte abgestützt wird.

Ein Schwerpunkt bei der Nebelkartierung liegt bei der räumlichen Nebelstruktur und weniger bei der exakten Angabe der Nebelhäufigkeit. Die Nebelhäufigkeit wird als Intervallangabe in der Karte erscheinen. In Anbetracht der großen lokalen Abweichungen, die der Nebel aufweisen kann, soll nicht eine Genauigkeit vorgetäuscht werden, die schwierig zu erreichen ist.

\subsection{Satellitendaten}

Die Information aus den Satellitenbildern lag aufbereitet in Matrixform vor. Pro Quadrat von $2 \mathrm{~km}$ Kantenlänge wurde für die ganze Schweiz ein Nebelbedeckungsgrad in Prozent der verwendeten Satellitenbilder berechnet. WANNER und KUNZ (1983) legten ihrer Arbeit ein Kollektiv von 94 Satellitenbildern zu Grunde. Bei der Auswahl der Satellitenbilder wurde eine gut erkennbare und geschlossene Nebeldecke im Mittelland vorausgesetzt. Deshalb blieben Ereignisse mit stärkerer Bewölkung (Hangnebel) unberücksichtigt, was eine geringere Häufigkeit mit zunehmender Höhe zur Folge hatte (Fig.1). Aus der Mittelkarte der Nebelbedeckung wurde nun für sämtliche 457 Stationen derjenige $2 \times 2-\mathrm{km}^{2}$-Nebelwert ausgewählt, in welchem die Beobachtungsstation liegt.

Figur 1 zeigt die Höhenverteilung der Nebelhäufigkeit von Stations- und Satellitendaten. Die beiden Kurven weisen recht große Differenzen auf. Folgende Gründe können als Erklärung dafür aufgeführt werden:

- Auswahlkriterium der Satellitenbilder; von hoher Bewölkung klar abgrenzbare Boden- oder Hochnebeldecke, Hangnebeldecke blieb unberücksichtigt.

- Überflug des Satelliten vor 10 UTC; vor allem bei stärkerer Einstrahlung kann sich die Nebeldecke gegenüber 06 UTC verändert haben.

- Satellit beobachtet von oben; sämtliche Stationen unter einer Hochnebeldecke liegen vom Satelliten aus gesehen ebenfalls im Nebel.

- Nicht identische Beobachtungsreihen; Zeitraum der Satellitenbilder 1973/74-1980/81, 5jährige Beobachtungsreihe 1970/71-1974/75.

- für einige Höhenniveaus nur wenige Stationen.

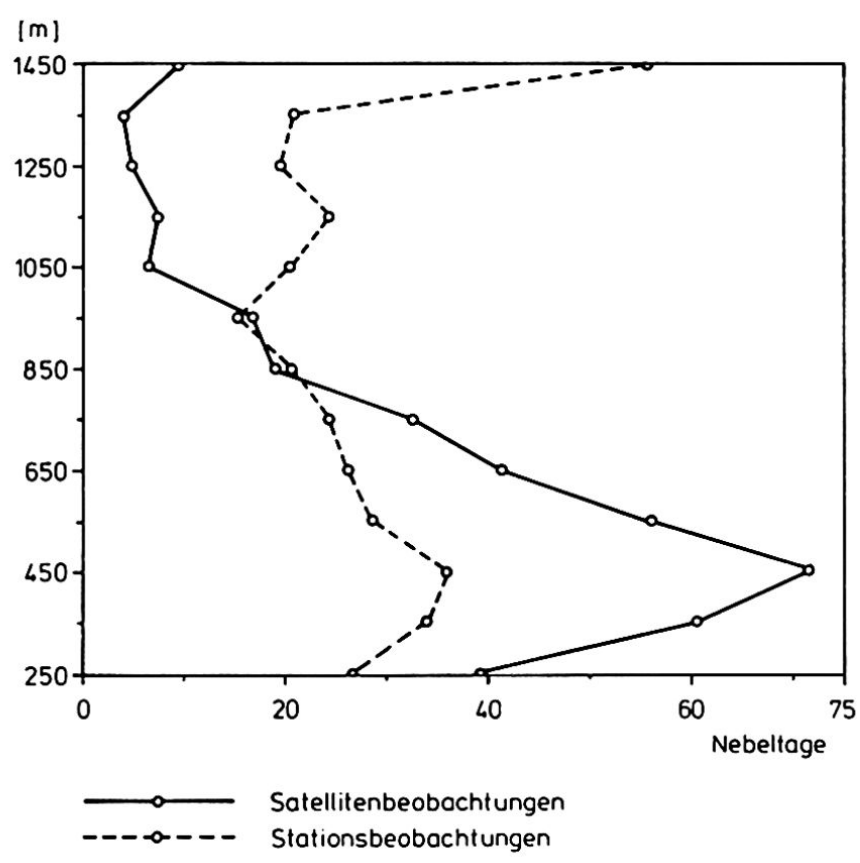

Fig.1 Höhenverteilung der mittleren Nebelhäufigkeit auf der Alpennordseite. Für Intervalle von 100 zu 100 m wurde der Stations- und Satellitenwert dargestellt. 


\section{Verbindung von Stations- und Satellitendaten}

Die hier beschriebene Methode zur Kombination von Stations- und Satellitendaten ist auf die Alpennordseite anwendbar. Die räumliche Einschränkung ergab sich aus dem Datenmaterial. Dieses erlaubte nicht, die gesamte Schweiz mit derselben Methode zu erfassen. Zusätzlich zur horizontalen Einschränkung mußte der Untersuchungsraum noch vertikal eingeengt werden. Über $1500 \mathrm{~m}$ ü. M. lieferte der Satellit keine relevanten Angaben mehr (Satellitenbildauswahl). In einem Flußdiagramm (Fig. 2) sind die einzelnen Arbeitsschritte für die Schätzung der Nebelhäufigkeit eines beliebigen Punkts im Untersuchungsraum aufgeführt. Bevor die beiden Datenquellen miteinander verknüpft wurden, sind fehlende Werte der 5jährigen Reihe ergänzt worden. Die Verknüpfung erfolgte sodann mit Hilfe einer Regression, in der die Stationswerte $\left(Y_{1}\right)$ in Abhängigkeit der Satellitenwerte $\left(X_{1}\right)$ geschätzt wurden. So ergab sich im Modell "Schätzwert 1». Anschließend wurden die Residuen (RESIJUAL $=Y_{2}$ ) berechnet und in Abhängigkeit der Höhe $\left(\mathrm{X}_{2}\right)$ dargestellt. Daraus ergab sich die zweite Modellgleichung und somit «Schätzwert 2».

Die Summe aus Schätzwert 1 und Schätzwert 2 ist in Figur 3 dargestellt. Für jeden Punkt der Alpennordseite unter $1500 \mathrm{~m}$ kann nun eine berechnete Nebelhäufigkeit angegeben werden. Mit diesem Verfahren wurde es möglich, aus Stationsbeobachtungen und Satellitendaten ei-

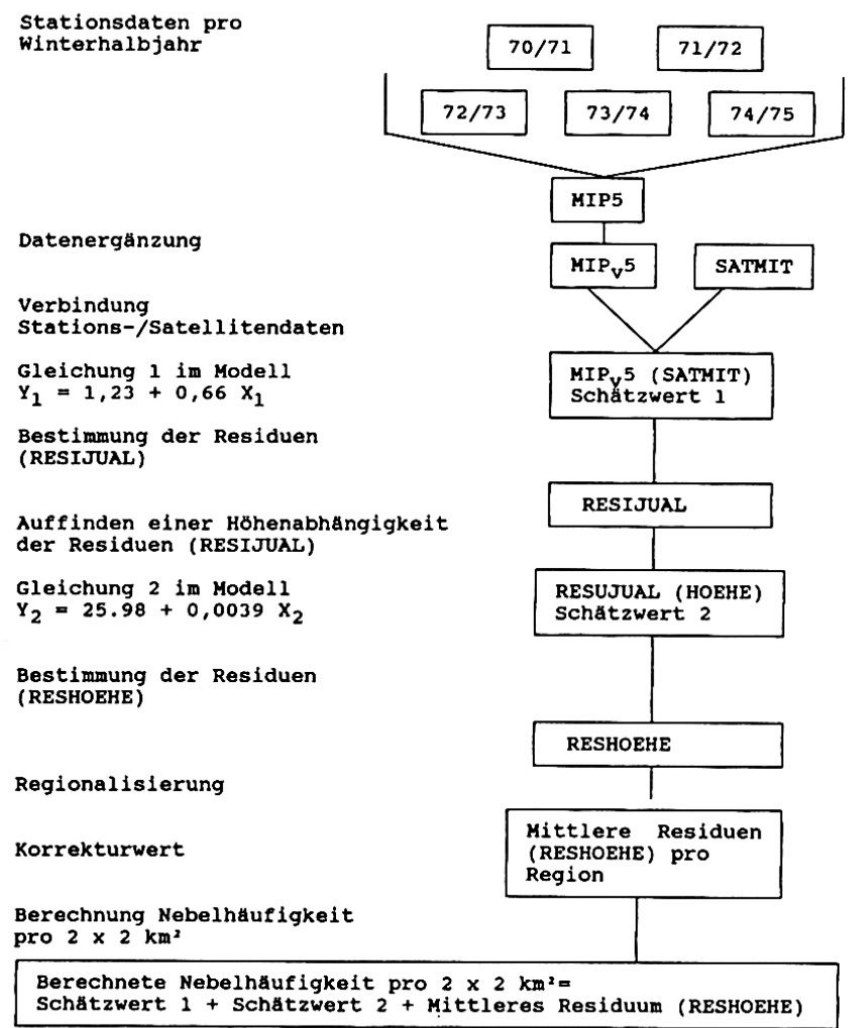

Fig.2 Flußdiagramm der Arbeitsschritte bei der Nebelkartierung. ne kombinierte, flächendeckende Aussage der Nebelhäufigkeit herzuleiten.

Die in Figur 3 berechneten Nebelhäufigkeiten mußten noch korrigiert werden. Mittels einer Klassierung der Residuen (RESHOEHE) war es möglich, eine Regionalisierung zu erhalten. Pro Region konnte sodann ein Korrekturwert (Mittelwert der Residuen pro Region) berechnet werden. Die Gleichung zur Schätzung der Nebelhäufigkeit beinhaltet demnach die folgenden Terme:

"Schätzwert 1»+ "Schätzwert 2" + "Mittleres Residuum»

\section{Ergebnis}

Das Resultat der Modellberechnungen wird in Figur 4 mit den effektiven Nebelhäufigkeiten verglichen. Dabei kann erkannt werden, daß sich die markanten Zonen der Nebelvorkommen auch bei der Höhenverteilung im Modell zeigen:

- Bodennebelmaximum auf

$450 \mathrm{~m}$ ü. $\mathrm{M}$.

- Nebelminimum auf

- Zunahme der Nebelhäufigkeit ab $1150 \mathrm{~m}$ ü. M. Weniger deutlich kommt im Modell die Hochnebelzone (ca. $750 \mathrm{~m}$ ü. M.) zum Ausdruck. Trotzdem ist erstaunlich, zu welchem befriedigenden Resultat die theoretische Berechnung der Nebelhäufigkeit führte, obwohl nur zwei Inputgrößen (Satellitenwert, Höhe über Meer), verwendet wurden. Offenbar genügen diese beiden Größen, um zu einer mittleren Verteilung der Nebelhäufigkeit zu gelangen.

Nach diesen eher theoretischen Resultaten sei auf das eigentliche Produkt dieser Nebeldatenauswertung hingewiesen. Auf Grund des befriedigenden Resultats der Modellrechnung stand nun pro $2 \times 2 \mathrm{~km}^{2}$ ein Nebelwert zur Verfügung. Diese Datendichte erlaubte, eine recht differenzierte Karte der Nebelverteilung zu erstellen (Fig. 6).

\section{Kartierung der Nebelstruktur und Nebelhäufigkeit im Gebiet der Schweiz}

Die Umsetzung des Resultates (Kap.4) in eine Nebelkarte Schweiz mit Angabe der Nebelstruktur und -häufigkeit erfolgte im Maßstab 1:200000. Entsprechend ihrem räumlichen Auftreten werden in der Karte drei Nebelarten ausgeschieden: Bodennebel, Hochnebel und Hangnebel. Um diese Nebelarten abgrenzen zu können, braucht es Angaben über die jeweiligen Höhenlagen der Nebelunter- und -obergrenzen. Für das Auffinden dieser markanten Nebelgrenzen wurden die Nebelwerte in Abhängigkeit der Meereshöhe dargestellt (Fig.7).

1. In den tiefsten Regionen unseres Landes führen die häufigen Bodennebel zu einem Nebelmaximum. Diese Bodennebelzone (1) steigt in der Westschweiz bis 


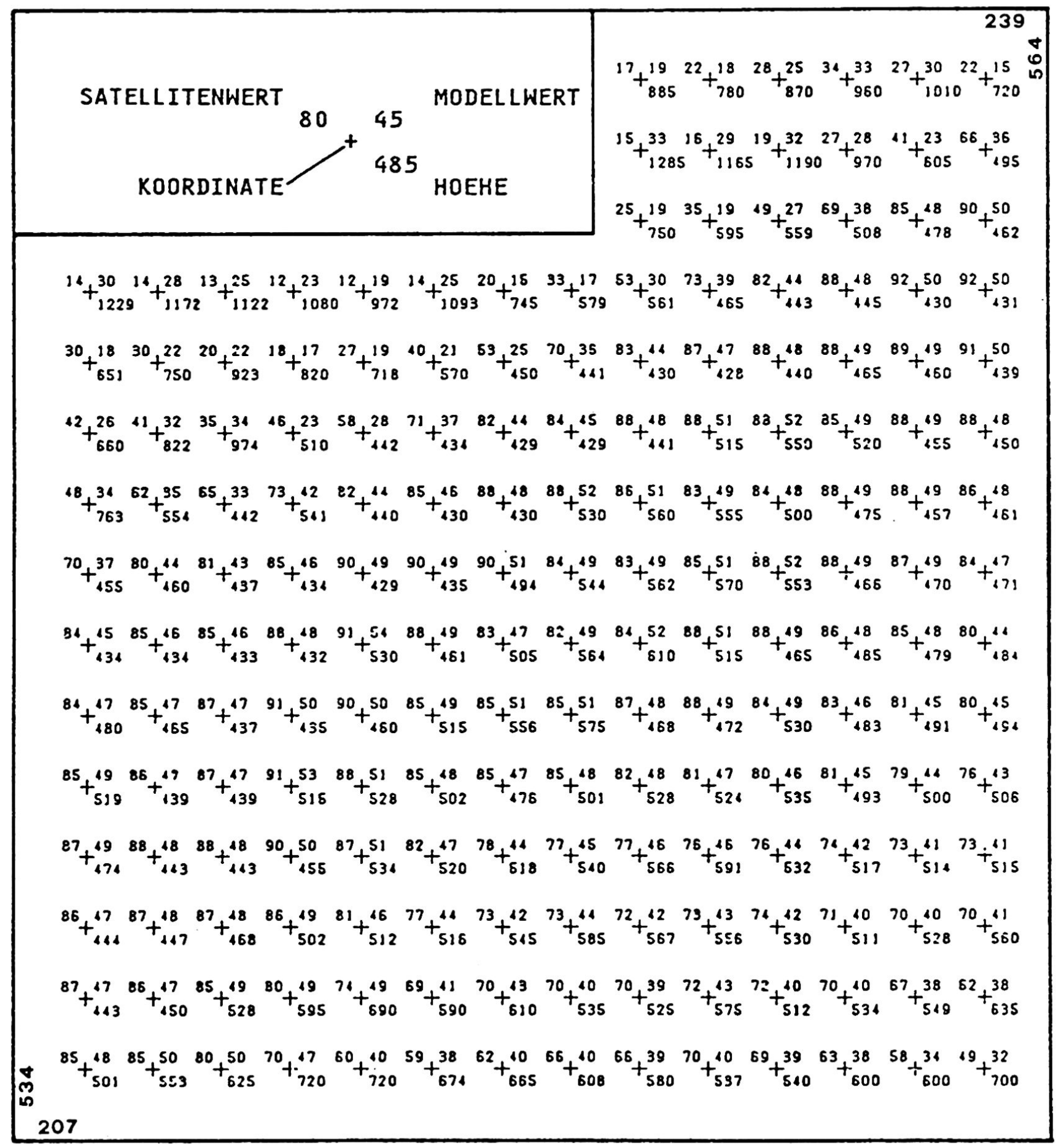

Fig.3 Geschätzte Nebelhäufigkeit pro $2 \times 2$ km²: "Schätzwert 1 + Schätzwert 2" 
[m]



$\longrightarrow$ Modell

- $-\infty-\ldots$ Realität

Fig.4 Mitteldarstellung der Nebelhäufigkeit. Vergleich Realität/Modell.

$650 \mathrm{~m}$ ü. M. an gegenüber $550 \mathrm{~m}$ ü. M. in der Ostschweiz.

2. Darüber folgt eine Zone mit weniger Nebel, die als nebelarme Zwischenzone bezeichnet wird. Jene Zone charakterisiert das Gebiet, welches zeitweise über dem Bodennebel oder unter dem Hochnebel liegt. Man beachte auch hier die Ost-West-Differenzierung von $100 \mathrm{~m}$.

3. Im Bereich von $750 \mathrm{~m}$ ü. M. (Ostschweiz) und $850 \mathrm{~m}$ ü.M. (Westschweiz) ist ein Anstieg der Nebelhäufigkeit zu beobachten. Dieser Nebelbereich kann der Hochnebelzone (3) zugerechnet werden. Der Höhenunterschied ergibt sich aus der Reliefverengung im Raum Genf. Der Alpenbogen und die Jurakette stoßen hier zusammen, und dies führt bei winterlichen Bisenlagen zu einem Anheben der Nebeldecke. Genetisch gesehen handelt es sich beim Boden- und Hochnebel fast ausschließlich um Strahlungs-Advektionsnebel.

4. Über der Hochnebelzone folgt die eigentliche Nebelminimumzone. Sie läßt sich eindeutig auf der Höhe von $950 \mathrm{~m}$ ü. M. ansetzen und wird als nebelarme Hangzone (4) bezeichnet.

5. Mit zunehmender Höhe folgt ein erneuter Anstieg der Nebelhäufigkeit. Diese Zone, die Hangnebelzone (5), repräsentiert die wolkenverhangenen Gebirgsregionen. Der Hangnebel wird vor allem bei zyklonalem Wetter auftreten. Er kann als Mischungsnebel, als orographischer Nebel, oder als Frontnebel vorkommen.
Häufig wird für diese Nebelart auch der Begriff «Schlechtwetternebel» verwendet.

Die Lokalisierung der markanten Nebelgrenzen in der Karte $1: 200000$ erforderte einige Interpretationen. Man denke an das Auslaufen einer Nebeldecke gegen die Alpentäler zu. Die ganze Kartierung wurde auch dadurch erschwert, daß der Nebel im Gegensatz zu anderen Wetterelementen (z. B. Druck, Temperatur) mit der Höhe einen nicht-stetigen Verlauf aufweist. Die Sprungschichten können regional und lokal sehr stark variieren. So entstand eine Kartengrundlage $1: 200000$ (Nebelart und Nebelhäufigkeit) für den Klimaatlas der Schweiz. Für diese Arbeit wurde die Nebelkarte Schweiz (Fig. 6) auf einen kleineren Maßstab umgezeichnet. Aus kartographischen Überlegungen wurden die nebelarme Zwischenzone und die Hochnebelzone zu einer einzigen Nebelzone zusammengefaßt. In der Nebelkarte kommt so die Gliederung in vier Nebelbereiche klar zum Ausdruck. Das Mittellandbecken tritt als Kaltluftsammeltrog (große Bodennebelhäufigkeit) deutlich hervor. Die nebelreichsten Gebiete finden sich entlang des JurasüdfuBes (Solothurn-Biel-Yverdon), im zentralen Mittelland (Aarau-Muri AG-Beznau) sowie in der Ostschweiz (Winterthur-Schaffhausen-Weinfelden). Diese lehnt sich mehrheitlich dem Gewässernetz der großen Mittellandflüsse an. Die größeren Juralängstäler weisen ebenfalls eine recht hohe Nebelhäufigkeit auf, währenddem die Hänge und die Jurahöhen vielfach verschont bleiben. Das Nebelgeschehen der N/NW-S/SE verlaufenden Täler im Übergang Mittelland-Alpen wird durch die Mächtigkeit der Nebeldecken im Mittelland geprägt. Die Bodennebelhäufigkeit verringert sich im allgemeinen gegen den Alpenrand zu. Trockentäler im Wallis und im Bündnerland haben selten Nebel. In den Tieflagen der Südschweiz können nur vereinzelt Bodennebelereignisse beobachtet werden.

Das reich gegliederte und daher auch wettermäßig stark differenzierte Gebiet der Schweiz beeinflußt die Nebelverteilung wesentlich. Die Häufigkeit der herbstlichen und der winterlichen Strahlungsnebel nimmt von einer gewissen Höhe über dem Tal stark ab. Je nach der Höhenlage und der Morphologie, welche die Mächtigkeit von Inversionen mitbestimmt, befindet sich die nebelarme Zwischenzone auf unterschiedlicher Meereshöhe. Einzelne Jurahöhen sowie das Voralpengebiet weisen wieder eine größere Anzahl Nebeltage auf. Diese Gebiete befinden sich vor allem in der Hochnebelzone. Die Nebelminimumzone folgt in der ganzen Schweiz oberhalb der Hochnebelgrenze. Hier treten im Mittel weniger als 10 Nebeltage pro Winterhalbjahr auf. Die untere Hangnebelzone verdeutlicht den erneuten Anstieg der Nebelhäufigkeit. Genetisch betrachtet handelt es sich beim Hangnebel um Frost-, Mischungs- oder orographische Nebel. Mangels Daten konnte das Gebiet der Schweiz nur bis $2000 \mathrm{~m}$ ü. M. ausgewertet werden. Im übrigen Gebiet sind noch einige Gebirgsstationen eingetragen (Arosa, Grimsel, Jungfraujoch, Weißfluhjoch), welche die markante Zunahme des Schlechtwetternebels mit der Höhe zeigen. 


\section{MITTELKARTEN DER NEBELBEDECKUNG}

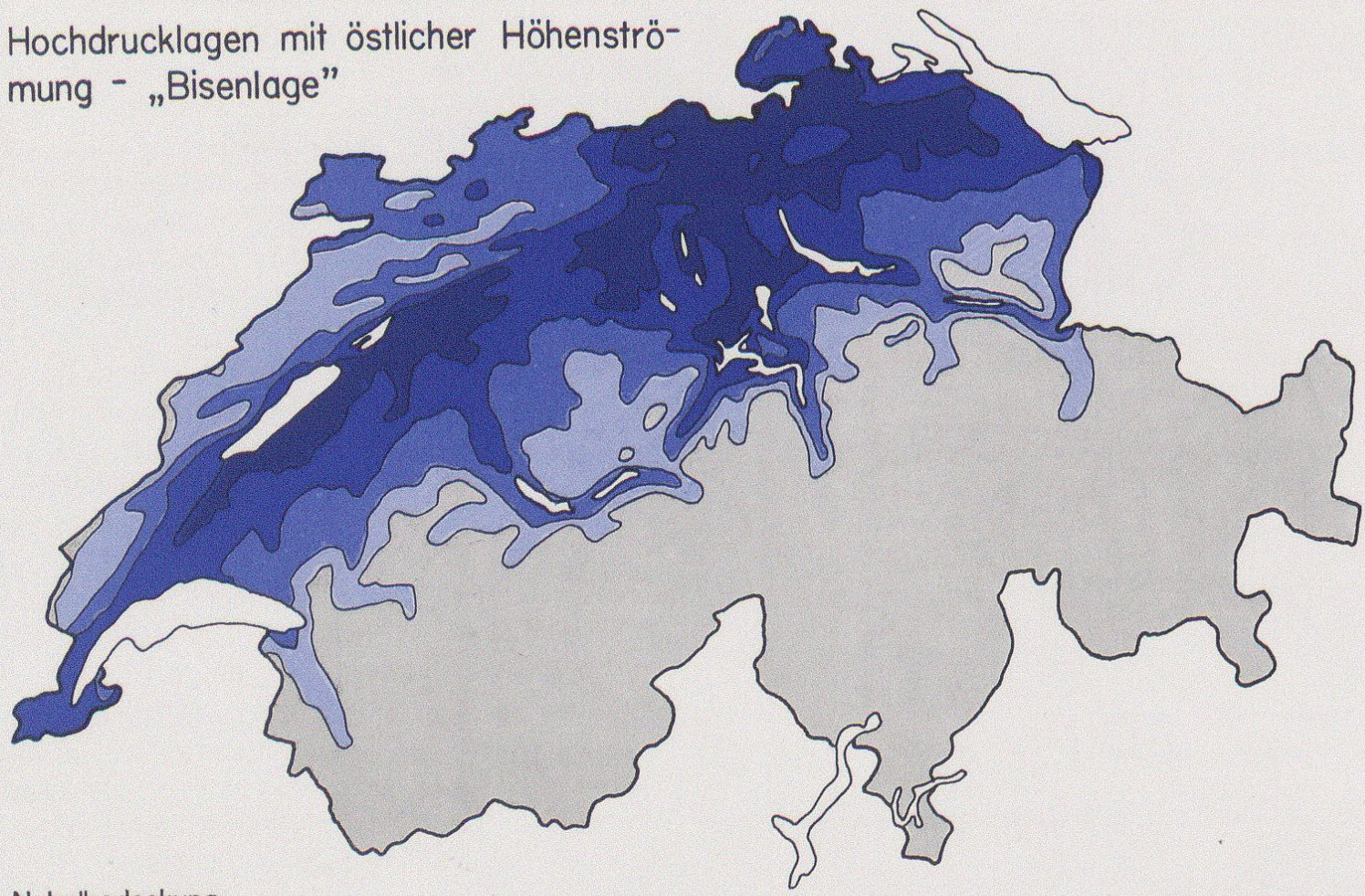

Nebelbedeckung

Fig. 5a Mittlere Nebelbedeckung bei Hochdrucklagen mit östlicher Höhenströmung - «Bisenlage».

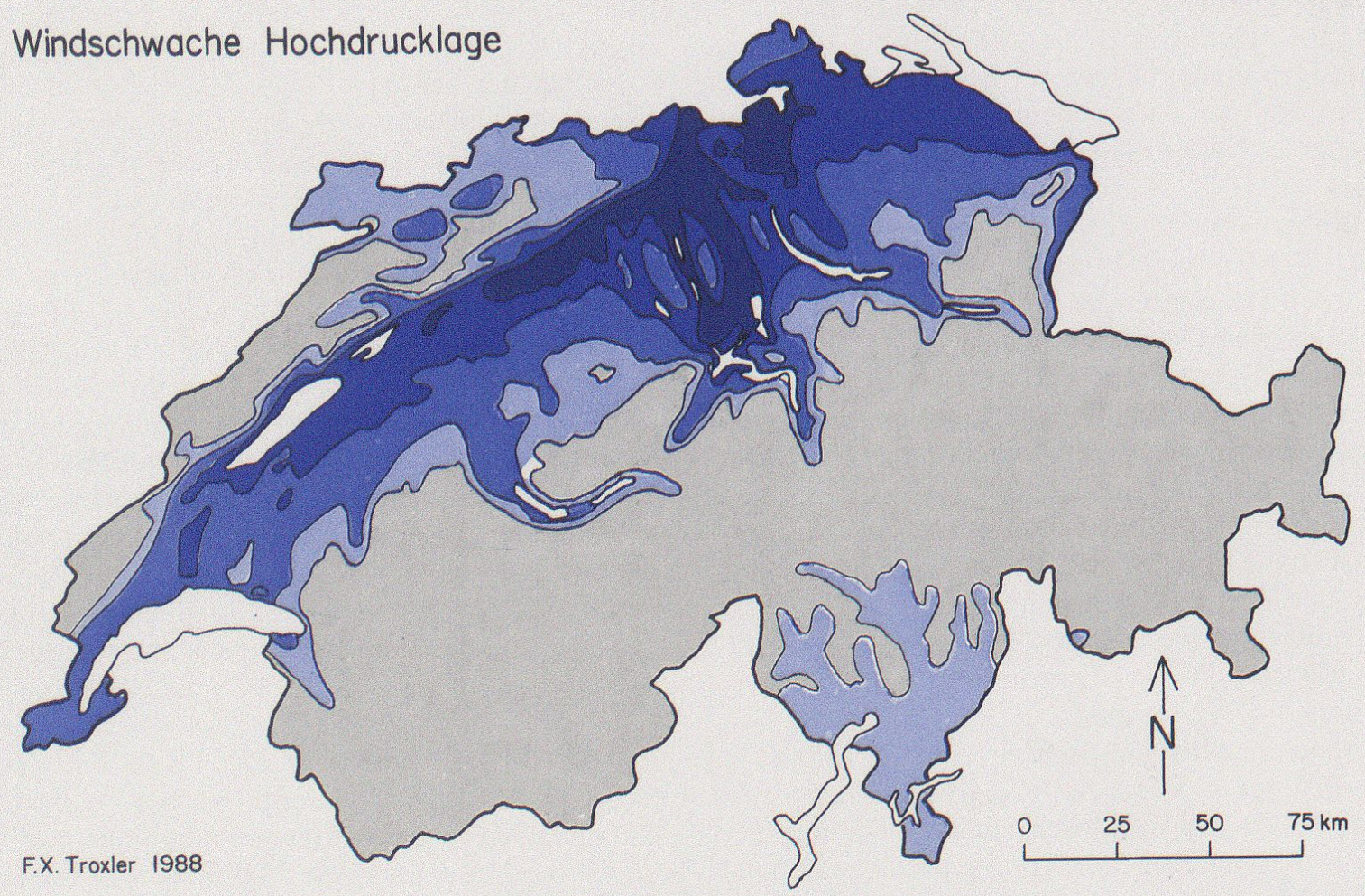

Fig.5b Mittlere Nebelbedeckung bei windschwachen Hochdrucklagen. 


\section{MITTELKARTEN DER NEBELBEDECKUNG}

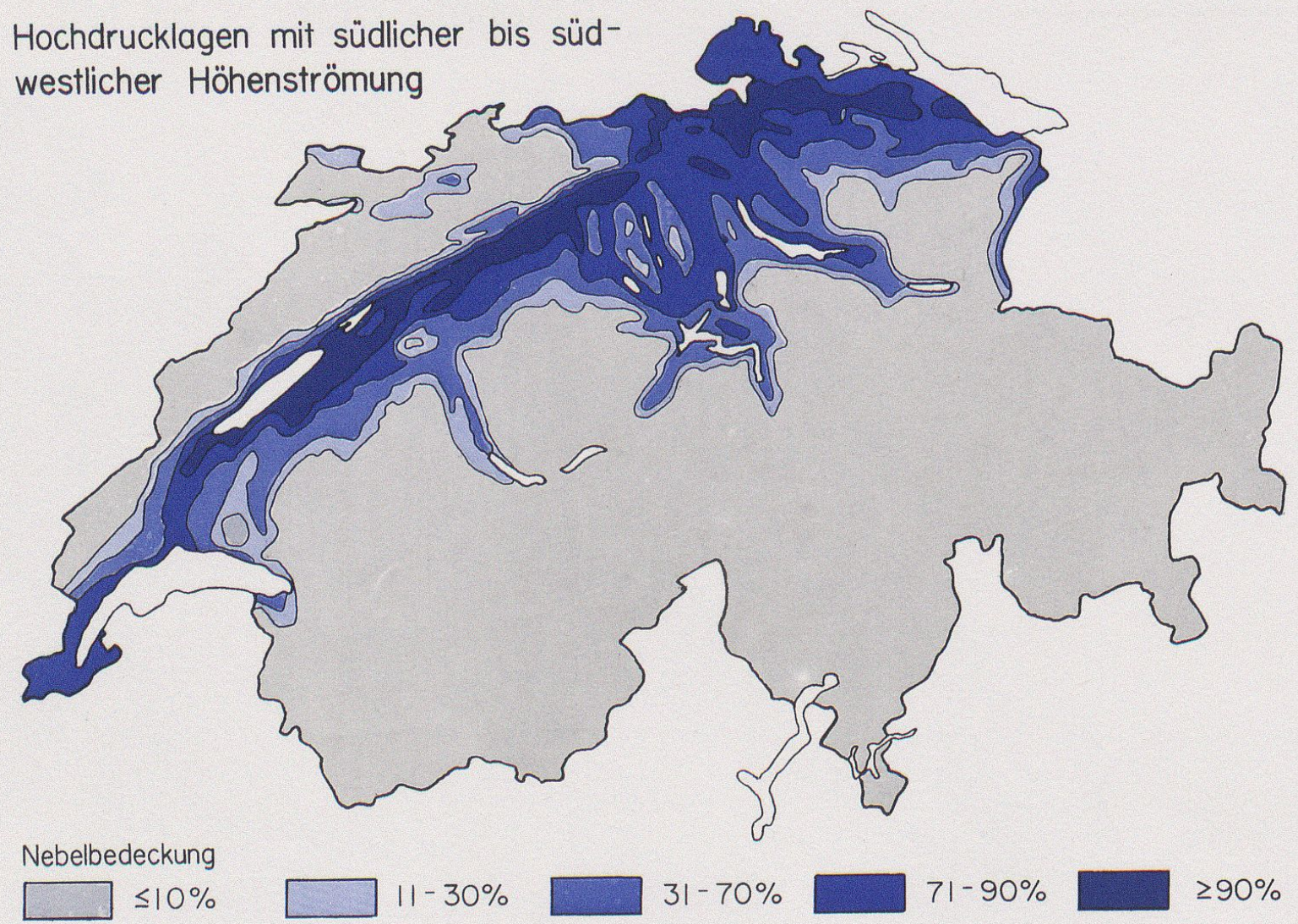

Fig.5c Mittlere Nebelbedeckung bei Hochdrucklagen mit südlicher bis südwestlicher Höhenströmung.

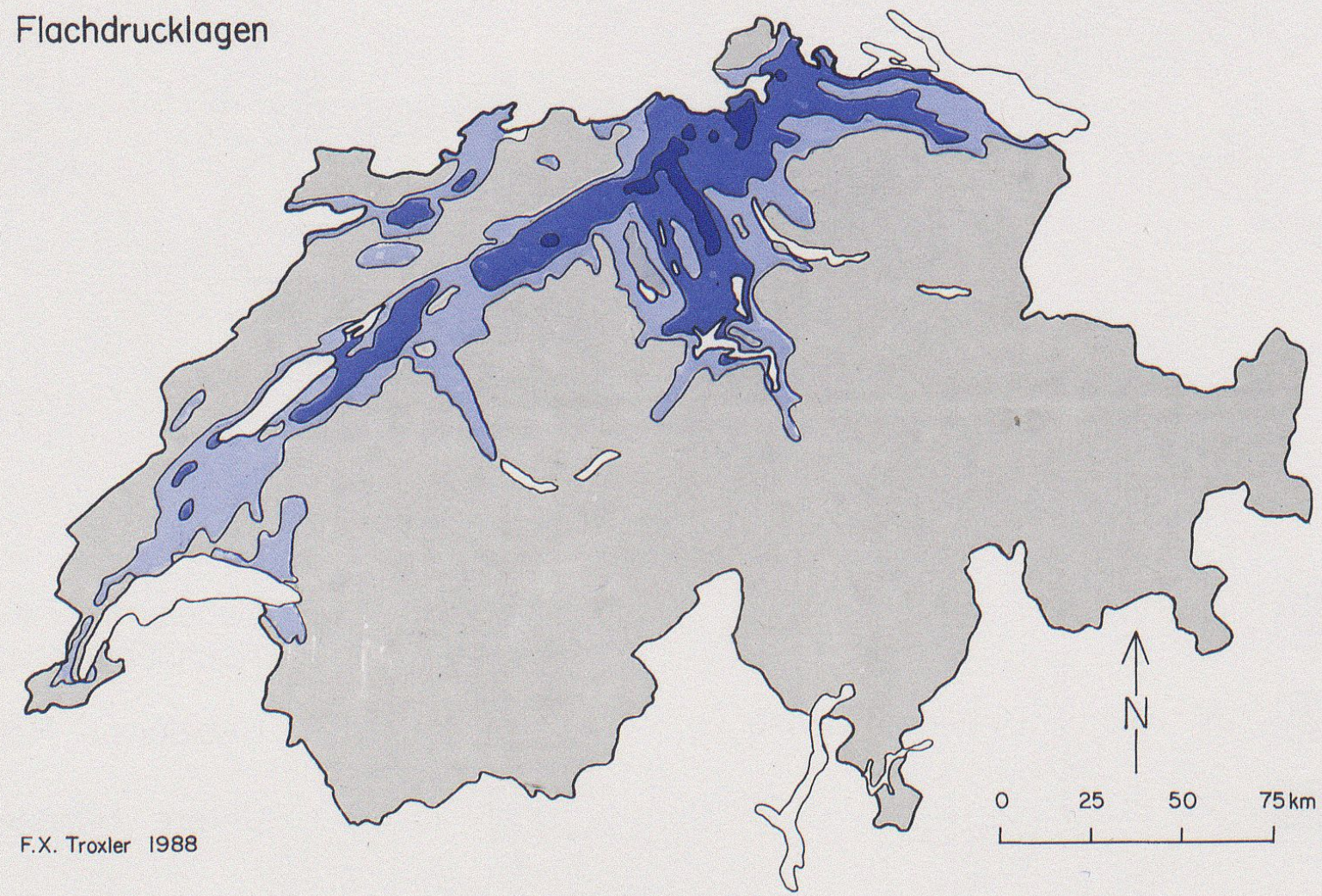

Fig.5d Mittlere Nebelbedeckung bei Flachdrucklagen. 


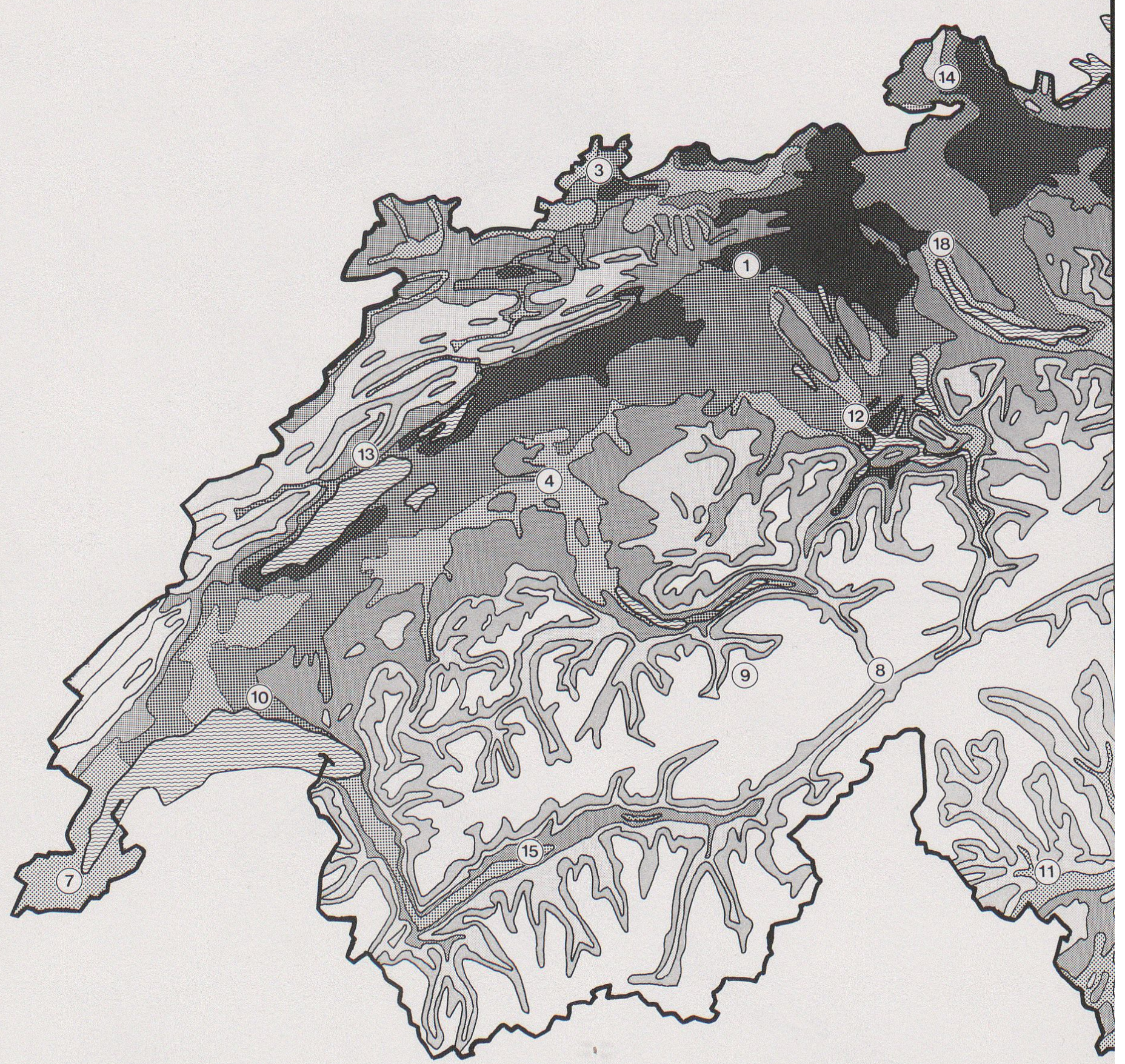

Nebeltage



Nebeltage

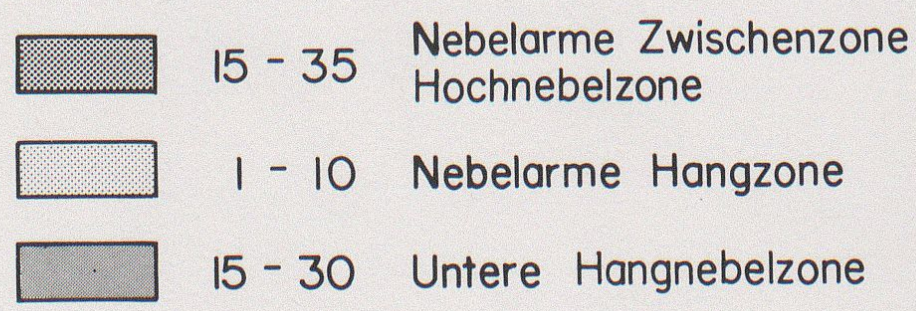

Fig.6 Nebelkarte. Art und Häufigkeit des Morgennebels, Mitteldarstellung der Winterhalbjahre 1970/71 bis 1974/75, Monate Oktober bis März, Beobachtung zwischen 7.00 und 8.00 Uhr. 
litteldarstellung der Winterhalbjahre 1970/71 bis $1974 / 75$, ig zwischen 07.00 und $08.00 \mathrm{Uhr}$.

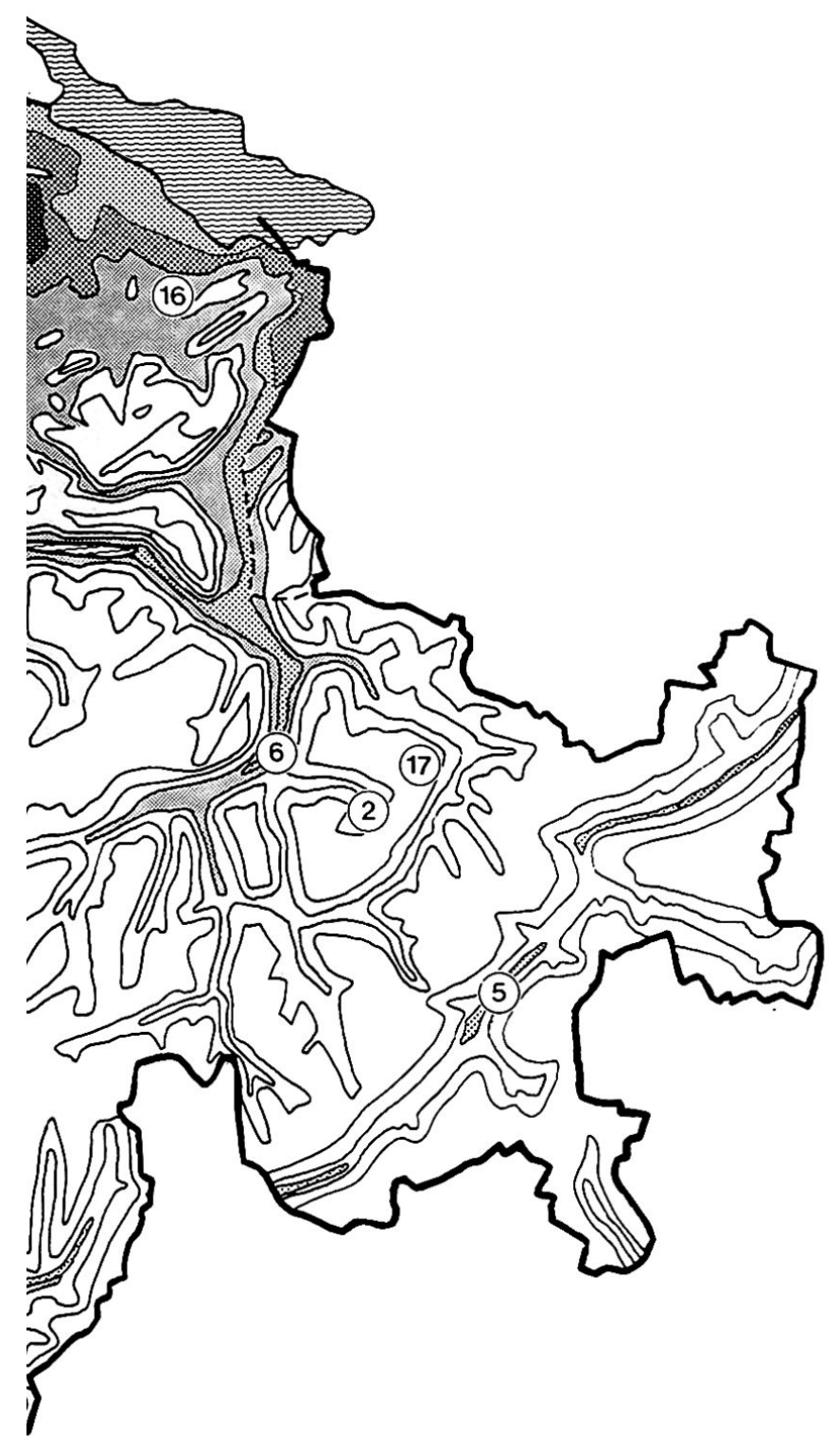

Stationsname Stationshöhe Nebeltage

$\begin{array}{lrr}\text { I. Aarau } & 409 \mathrm{~m} & 60,8 \\ \text { 2. Arosa } & 1821 \mathrm{~m} & 65,0 \\ \text { 3. Basel } & 316 \mathrm{~m} & 27,4 \\ \text { 4. Bern } & 560 \mathrm{~m} & 21,6 \\ \text { 5. Bever } & 1710 \mathrm{~m} & 19,2 \\ \text { 6. Chur } & 582 \mathrm{~m} & 13,4 \\ \text { 7. Genf } & 420 \mathrm{~m} & 29,6 \\ \text { 8. Grimsel } & 1950 \mathrm{~m} & 34,2 \\ \text { 9. Jungfraujoch } & 3572 \mathrm{~m} & 63,8 \\ \text { 10. Lausanne } & 605 \mathrm{~m} & 40,4 \\ \text { 11. Locarno } & 366 \mathrm{~m} & 30,2 \\ \text { 12. Luzern } & 456 \mathrm{~m} & 49,8 \\ \text { 13. Neuenburg } & 485 \mathrm{~m} & 27,0 \\ \text { 14. Schaffhausen } & 435 \mathrm{~m} & 51,4 \\ \text { 15. Sitten } & 542 \mathrm{~m} & 11,8 \\ \text { 16. St. Gallen } & 670 \mathrm{~m} & 65,4 \\ \text { 17. Weissfluhjoch } & 2672 \mathrm{~m} & 84,6 \\ \text { 18. Zürich } & 556 \mathrm{~m} & 32,6\end{array}$



$1: 1500000$

Gebiet über 2000 mü.M.

Seen

F.X. TROXLER 1988 
[m]

Westschweiz



[m] Ostschweiz

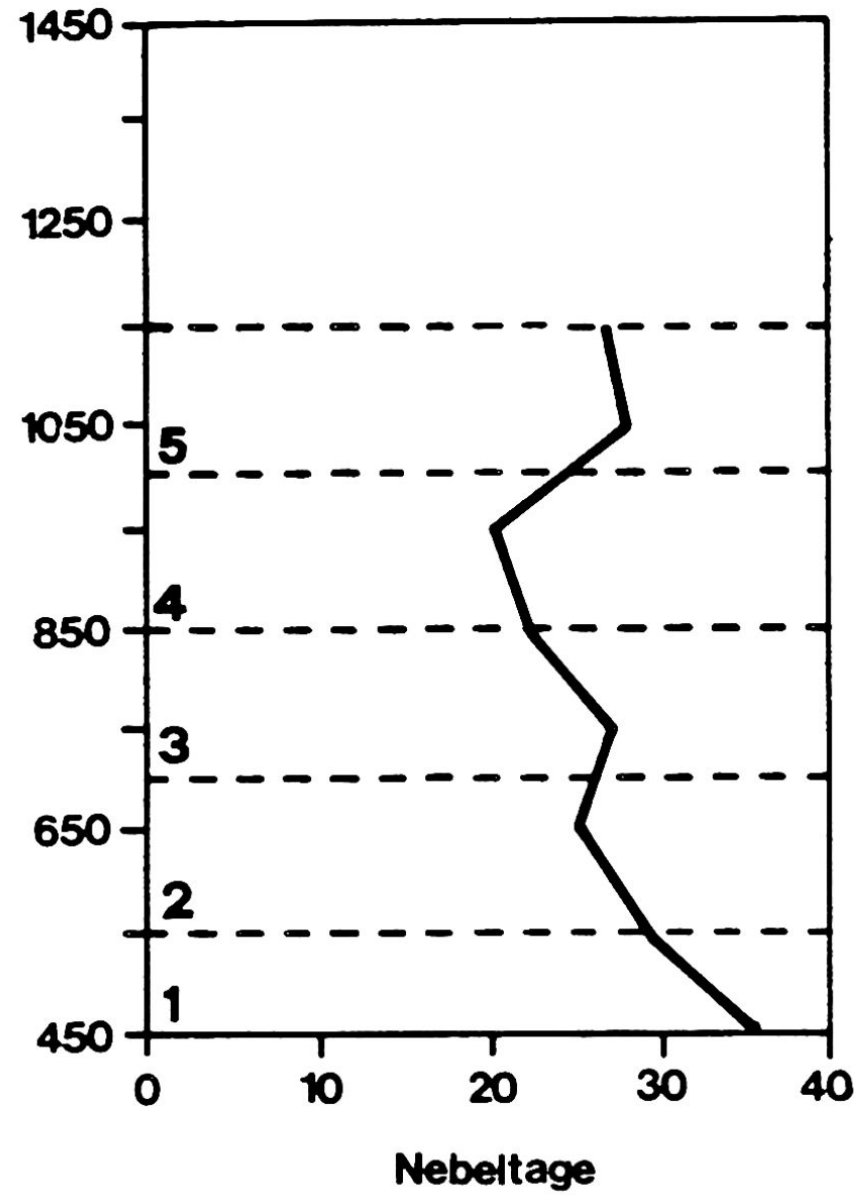

Fig.7 Höhenverteilung der Nebelhäufigkeit, aufgeteilt in West- und Ostschweiz, für die Winterhalbjahre 1970/71-1974-75 (Monate Oktober bis März). Für die Intervalle von 100 zu 100 m wurde der Mittelwert dargestellt.

6. Die Nebelverteilung in Abhängigkeit verschiedener Wetterlagen und ihre Auswirkungen auf die Durchlüftung

In diesem Kapitel sollen die Resultate der Nebelauswertungen, welche mit Hilfe von Satellitenbildern durchgeführt wurden, dargestellt werden. Längere Reihen von Wettersatellitenbildern erlauben heute eine objektive Kartierung der Nebelbedeckung zu einem bestimmten Zeitpunkt. Das Gesamtkollektiv der nach Wetterlage aufgeschlüsselten Daten wurde wie erwähnt als Mittelwertskarte der Nebelbedeckung für die Basiskartierung verwendet.

In ihrer Arbeit haben WANNER und KUNZ (1983) verschiedene wetterlagenabhängige Mittelkarten der Nebelbedeckung entworfen und beschrieben. Für ihre Berechnungen haben sie 94 Satellitenbilder mit einer typischen Nebelverteilung ausgewählt. Die für die Nebelverteilung (Durchlüftung) relevanten Wetterlagen werden hier kurz erläutert. Für jede Wetterlage wird zudem die Nebelverteilung in einer Karte (Fig. 5a-d) dargestellt.

\section{a) Hochdrucklage mit östlicher Höhenströmung ("Bisenlage»)}

Abgesehen vom Druckanstieg, von der Bewölkungsabnahme und der zunehmenden Ausstrahlung sind die herbstlichen Hochdrucklagen in der Initialphase (d.h. nach Kaltfrontdurchgängen) auf der Alpennordseite sehr oft durch leichte Ostwinde mit Kaltluftadvektion gekennzeichnet. Dieser Effekt wird mit der Abkühlung der eurasiatischen Landmasse und der Verstärkung der sibirischen Bodenantizyklone im Verlaufe des Winters immer deutlicher. Die Nebelverteilung deutet darauf hin, daß sich bei östlichen Strömungen aufder Alpennordseite ein Stau der Kaltluft einstellt, der im Schweizer Mittelland durch die Konvergenz von Jura und Alpen bei Genf zusätzlich akzentuiert wird. Durch die Zunahme der mechanischen Turbulenz innerhalb der ABL (Atmospheric Boundary Layer), und durch den erwähnten Stau wird die Obergrenze der Kaltluft und Nebeldecke zum Teil massiv angehoben, und weite Teile des Schweizer Mittellandes, aber auch der Juranordseite (Raum Basel) und 
der Voralpen weisen eine hohe Nebelbedeckungsrate auf. Regelmäßig auftretende Wiederanstiege der Nebelobergrenze während Hochdruckperioden sind auf «Bisenrückfälle» mit erhöhter Turbulenz zurückzuführen (Fig. 5a).

\section{b) Windschwache Hochdrucklagen}

Die im Zuge solcher Hochdruckepisoden häufig beobachtete Verlagerung des Hochdruckkerns in den Alpenraum, die damit verbundene Abnahme mechanisch induzierter Turbulenz sowie die zunehmene Subsidenz führen im Schweizer Mittelland zu einem Absinken der Nebelobergrenze bis auf eine Gleichgewichtshöhe von ca. $750 \mathrm{~m}$ ü. M. Bei dieser Wetterlage weisen die Gebiete nördlich des Jura eine tiefere, südlich der Alpen hingegen eine höhere Bedeckungshäufigkeit auf als bei der Bisenlage (Fig. 5b).

\section{c) Hochdrucklagen mit südlicher oder südwestlicher Höhenströmung}

Beim Übergang zu südlichen Strömungsrichtungen, d.h. in der Schlußphase von Nebelperioden (Abbau des Hochs über den Alpen von Westen her), sinkt die Nebelobergrenze des Schweizer Mittellandes in der Regel stark ab (500-600 m ü. M.) Das Absinken wird durch das Ausfließen der Kaltluft Richtung NE (Bodensee) und vor allem Richtung Hochrhein-Basel bewirkt. Zusätzlich dürfte auch die Scherungsturbulenz im Bereich zwischen der bodennahen Kaltluft und der aufgleitenden Südwestströmung eine Rolle spielen. Dieser Effekt kann sich infolge der abnehmenden Stabilität innerhalb der ABL dauernd verstärken. Die Nebelgefährdung des Mittellandes beschränkt sich bei dieser Wetterlage sehr oft auf ein schmales Kaltluftband am Jurasüdfuß, welches dann ausgesprochen durchlüftungsarme Verhältnisse aufweist (Fig. 5c).

\section{d) Flachdrucklagen im Frühherbst und Frühling}

Die Bildung und Auflösung der Kaltluft- und Nebelkörper ist ganz erheblich von der Energiebilanz abhängig. Im Frühherbst und Frühling erreicht die Nebelobergrenze bei «Schönwetter» (flache Druckverteilung, geringe Turbulenz) kaum die Höhe von 750 m ü. M. Bei dieser Wetterlage treten die eigentlichen Kernzonen der Nebelgebiete (Jurasüdfuß, Reußtal) besonders hervor. Da es sich in diesem Fall in erster Linie um Strahlungsnebel handelt, kommen durch diese Nebelverteilung die Gebiete mit extremer Kaltluftgefährdung zum Vorschein. Es sind dies auch Gebiete im Voralpengebiet mit stark reduziertem Luftaustausch (Fig. 5d).
Wie schon in der Einleitung (Kap.1) angetönt wurde, kommt der Anwesenheit von Wasserdampf in der Atmosphäre aus lufthygienischer Sicht insofern eine große Bedeutung zu, als in der wäßrigen Phase und vor allem im Wechsel mit gasförmigen Prozessen sehr komplexe chemische Reaktionen ablaufen.

Nebellagen zeichnen sich durch eine stabile Schichtung im Bereich der Nebelobergrenze sowie durch Schwachwinde aus. Beide Phänomene schränken die Verdünnung von Schadstoffen ein. Wie aus WANNER und KUNZ (1983) hervorgeht, ist die Häufigkeit einer Inversion ohne Nebel im Schweizer Mittelland vier- bis sechsmal größer als jene des Auftretens von Morgennebel. Das bedeutet nichts anderes, als daß im Schweizer Mittelland eine stabile Grenzschicht ohne Nebel häufiger auftritt als eine solche mit Nebel.

\section{Abkïrzungen:}

MIP5 Mittlere Anzahl Nebeltage pro Station der unvollständigen 5jährigen Beobachtungsreihe 1970/71-1974/75

MIP 5: $\quad$ Mittlere Anzahl Nebeltage der vollständigen 5jährigen Beobachtungsreihe 1970/ 71-1974/75

SATMIT: Mittlere Nebelbedeckung in \% der verwendeten Satellitenbilder, und zwar jener $2 \times 2 \mathrm{~km}^{2}$ Rasterwert, in dem sich die Station befindet

RESIJUAL: Residuen aus der Beziehung MIP 5 (SATMIT) für die Region Jura bis Nordalpenkamm; beobachtete minus theoretische Nebelhäufigkeit

RESHOEHE: Residuen aus der Beziehung RESUJUAL (HOEHE) für die Region Jura bis Nordalpenkamm; effektives minus theoretisch berechnetes Residuum

SMA: $\quad$ Schweizerische Meteorologische Anstalt

Die Arbeit wurde unterstützt durch den schweizerischen Nationalfonds zur Förderung der wissenschaftlichen Forschung (NFP 14).

\section{Literatur}

STUMM, W., SIGG, L., ZOBRIST, J. und JOHNSON, A. (1985): Nebel als Träger konzentrierter Schadstoffe. Neue Zürcher Zeitung, 16. Januar, Nr. 12, Zürich.

TROXLER, F.X. (1987): Nebelkartierung Schweiz. Stationsund Satellitendaten als Grundlage. Diplomarbeit Geogr. Institut Bern, $106 \mathrm{~S}$.

WANNER, H. und KUNZ, S. (1983): Klimatologie der Nebelund Kaltluftkörper im schweizerischen Alpenvorland mit Hilfe von Wettersatellitenbildern. Arch. Met. Geoph. Biocl. B33, 31-56. 